\title{
RISCOS OCUPACIONAIS À SAÚDE E VOZ DE PROFESSORES: ESPECIFICIDADES DAS UNIDADES DE REDE MUNICIPAL DE ENSINO
}

\section{Occupational risks to the health and voice of teachers: specificities of municipal education units}

\author{
Emilse Aparecida Merlin Servilha ${ }^{(1)}$, Isabela de Sousa Ruela ${ }^{(2)}$
}

\begin{abstract}
RESUMO
Objetivo: comparar as condições de trabalho, saúde e voz em professores de diferentes escolas municipais. Métodos: participaram 165 professores, média de 37 anos, casados, predomínio de mulheres e escolaridade superior da rede municipal de ensino de uma cidade do estado de São Paulo, que responderam ao questionário proposto por Ferreira et al (2003), abrangendo questões sócio-demográficas, situação funcional, aspectos vocais e de saúde. As respostas dos docentes foram separadas por unidade escolar e submetidas à análise estatística. Resultados: constatouse diferença significante entre as escolas quanto à presença de local para descanso, fiscalização constante e facilidade para se ausentar da sala de aula, número de alunos por classe $(<0,001)$, ruído $(0,002)$, iluminação $(<0,001)$, limpeza $(0,001)$, higiene nos sanitários $(0,002)$, poeira $(0,001)$, tamanho da sala $(0,020)$, suficiência de espaço em classe $(0,005)$, comprometimento dos funcionários com a manutenção da escola $(0,033)$ e temperatura $(<0,001)$. As menções de alteração vocal variaram de $76,47 \%$ a $40 \%$, sem significância nos professores das diferentes escolas. Os problemas de saúde foram diversificados com diferença significante apenas para incômodo a sons $(0,036)$. Conclusão: há riscos ocupacionais específicos relacionados à organização e ambiente de trabalho, nas distintas unidades escolares, que interferem na voz e saúde dos docentes, cujo desvelamento subsidia o gestor na implantação de ações contextualizadas, visando promover ambiente saudável para toda comunidade escolar.
\end{abstract}

DESCRITORES: Distúrbios da Voz; Docentes; Riscos Ocupacionais

\section{INTRODUÇÃO}

As condições ambientais inapropriadas das escolas quanto aos níveis de ruído, estado de limpeza, ventilação, iluminação e temperatura ${ }^{1-5}$, acrescidas à organização de trabalho insatisfatória com excesso de atividades, falta de momentos de descanso e excessiva fiscalização, prejudicam a saúde física e mental dos professores, além de provocarem alterações vocais ${ }^{6,7}$. Todos esses

(1) Fonoaudióloga; Docente da Faculdade de Fonoaudiologia da Pontifícia Universidade Católica de Campinas, PUCCAMP, Campinas, SP; Doutora em Ciências pela Pontifícia Universidade Católica de Campinas.

(2) Aluna do Curso de Graduação em Fonoaudiologia; Bolsista de Iniciação Científica PIBIC/CNPq 2007/2008.

Conflito de interesses: inexistente fatores ligados ao trabalho permitem considerar os professores como grupo de risco para os distúrbios vocais, com consequente absentismo, afastamento e até readaptação ao trabalho ${ }^{8}$.

A rouquidão, fadiga vocal, ardor na garganta, esforço ao falar, dificuldade em manter a intensidade, dificuldade em projetar a voz e afonia têm sido citados como os sinais e sintomas frequentes entre os professores ${ }^{9,10}$ com consequências negativas para o processo ensino-aprendizagem ${ }^{11}$.

Estudos epidemiológicos que investigam as relações entre saúde, trabalho e voz do professor mostram-se de interesse para a elaboração de políticas públicas que visem proteger a saúde do trabalhador, em especial a do professor. Ao mesmo tempo, faz-se necessário também particularizar o funcionamento das escolas e desvelar os riscos ocupacionais nelas presentes para sua 
minimização ou eliminação, com o intuito de salvaguardar as condições de salubridade para a convivência de professores e alunos. Nesse contexto, o objetivo desse estudo foi comparar as condições de trabalho, saúde e voz em professores de rede municipal de ensino, particularizando os riscos ocupacionais presentes nas diferentes unidades escolares.

\section{MÉTODOS}

Trata-se de estudo epidemiológico de corte transversal, do qual participaram 165 professores, média de 37 anos, predomínio mulheres, casados e escolaridade superior, da rede municipal de ensino de uma cidade do interior do estado de São Paulo, distribuídos em nove unidades escolares e englobando desde a Educação Infantil até a Educação de Jovens e Adultos (EJA). A partir da definição do número da amostra, utilizou-se o sorteio dos participantes, respeitando-se a proporção do número de professores e dos distintos níveis de ensino da rede municipal de educação.

Foi aplicado, em 2006, um questionário ${ }^{9}$ que incluía dados pessoais, situação funcional, aspectos de saúde vocal e geral, hábitos, antecedentes familiares e lazer dos participantes.

Estabeleceram-se como critérios de inclusão: ser professor da rede municipal de ensino, estar trabalhando e aceitar participar da pesquisa de forma livre e esclarecida; e como critérios de exclusão: recusa em participar da pesquisa, ausência ou estar afastado do trabalho no dia da avaliação.

A pesquisa foi aprovada pelo Comitê de Ética em Pesquisa institucional em 29/05/2007 com o protocolo $n^{\circ}$. 182/07.

As respostas dos professores foram organizadas, digitalizadas em programa Excel e os dados separados por unidade de ensino; em seguida, procedeu-se a análise descritiva de todas as variáveis. Para aquelas de caráter quantitativo procedeu-se a observação dos valores mínimos e máximos, e o cálculo de médias, desvios-padrão e mediana. Para as variáveis qualitativas calcularam-se frequências absolutas e relativas.

Para a comparação de médias dos grupos estudados foi utilizada a Análise de Variância a um fato. Quando a suposição de normalidade dos dados foi rejeitada foi utilizado o teste não-paramétrico de Kruskal-Wallis, com comparações múltiplas feitas através do teste de Dunn. Para se testar a homogeneidade entre as proporções foi utilizado o teste qui-quadrado ou o teste exato de Fisher, quando ocorreram frequências esperadas menores que $5{ }^{12}$. O nível de significância utilizado para os testes foi de $5 \%$.

\section{RESULTADOS}

Quanto aos dados demográficos não houve diferença significante entre as unidades escolares no que se refere à idade, estado civil, escolaridade e tempo de profissão, contudo, isto ocorreu em relação ao sexo $(0,003)$, uma vez que a unidade II mostrou uma porcentagem maior de homens que nas demais.

Quanto à situação funcional, as unidades diferem quanto ao nível de ensino $(<0,001)$ e isto repercute na faixa etária dos alunos $(<0,001)$.

A Tabela 1 mostra que na organização do trabaIho, o ponto comum entre as unidades foi o tempo curto para desenvolver as atividades na escola, variando de $90,91 \%$ a $60 \%$. Outros fatores mostraram porcentagens variáveis indicando ser relevante somente para algumas escolas. Houve diferença significante entre as escolas quanto à presença de local para descanso $(0,004)$, fiscalização constante $(0,017)$, e facilidade de se ausentar da sala de aula $(0,020)$. As demais comparações não apresentaram diferenças significantes.

No que tange às condições do ambiente físico (Tabela 2), as porcentagens das queixas também variaram muito, mostrando-se negativas em maior ou menor grau de acordo com a unidade. Assim, as escolas diferiram quanto ao número de alunos por classe $(<0,001)$, ruído $(0,002)$, iluminação $(0,001)$, limpeza $(0,001)$, condições de higiene nos sanitários $(0,002)$, poeira $(<0,001)$, tamanho da sala $(0,020)$, suficiência de espaço para locomoção em sala de aula $(0,005)$, comprometimento dos funcionários com a manutenção da escola $(0,033)$ e temperatura $(<0,001)$.

Quanto à voz (Tabela 3 ), as menções de alteração variaram de $76,47 \%$ a $40 \%$, atribuída principalmente ao uso intensivo de voz (100\% a $85,71 \%)$, stress $(100 \%$ a $50 \%)$ e alergia $(85,71 \%$ a $30 \%)$, no entanto a análise estatística não mostrou significância no quesito presença de alteração na voz nos professores e quanto à causa da mesma (Tabela 4), as unidades diferiram apenas em relação à infecção respiratória $(0,021)$.

Apesar da frequência e diversidade dos problemas de saúde (Tabela 5) mencionados pelos docentes, somente o incômodo a ruídos diferenciou as escolas $(0,036)$. 
Tabela 1 - Comparação entre as unidades nos quesitos relativos à organização de trabalho

\begin{tabular}{|c|c|c|c|c|c|c|c|c|c|}
\hline \multirow{2}{*}{ Aspectos da Organização de trabalho } & \multicolumn{8}{|c|}{ Unidades Escolares/ Dados em \% } & \multirow{2}{*}{ SE } \\
\hline & $\mathbf{I}$ & II & IV & $\mathbf{v}$ & VI & VII & VIII & IX & \\
\hline Possuir autonomia quanto ao planejamento & 91,18 & 100 & 100 & 100 & 94,74 & 85,71 & 100 & 92,68 & - \\
\hline Ritmo de trabalho estressante & 14,71 & 0 & 24 & 36,36 & 36,84 & 28,57 & 31,25 & 29,27 & - \\
\hline Material Adequado & 82,35 & 60 & 68 & 54,55 & 94,74 & 78,57 & 87,5 & 68,69 & - \\
\hline Tempo curto para desenvolver as atividades na escola & 82,35 & 60 & 64 & 90,91 & 89,47 & 85,71 & 81,25 & 90,24 & - \\
\hline Ambiente de trabalho - estressante & 20,59 & 0 & 20 & 9,09 & 31,58 & 50 & 0 & 24,39 & - \\
\hline Facilidade de se ausentar da sala de aula & 47 & 100 & 60 & 90,91 & 63,16 & 57,14 & 87,5 & 51,22 & 0,020 \\
\hline Presença de fiscalização do desempenho & 35,3 & 0 & 48 & 9 & 36,84 & 14,29 & 62,5 & 24,39 & 0,017 \\
\hline Presença de lugar para descanso na escola & 35,3 & 80 & 36 & 63,64 & 73,68 & 50 & 43,75 & 21,95 & 0,004 \\
\hline Carregar peso com frequência & 26,47 & 0 & 20 & 27,27 & 31,58 & 7,14 & 18,75 & 14,63 & - \\
\hline Realizar esforço físico & 14,71 & 0 & 16 & 27,27 & 10,53 & 7,14 & 18,75 & 26,83 & - \\
\hline
\end{tabular}

Legenda: SE - Significância estatística

Tabela 2 - Comparação entre as unidades quanto às condições do ambiente físico

\begin{tabular}{lccccccccc}
\hline \multirow{2}{*}{ Ambiente Físico } & \multicolumn{1}{c}{ Unidades Escolares/ Dados em \% } & \multicolumn{3}{c}{ SE } \\
\cline { 2 - 8 } & I & II & IV & V & VI & VII & VIII & IX & \\
\hline Iluminação adequada & 91,18 & 100 & 84 & 72,73 & 73,68 & 92,86 & 87,5 & 51,22 & 0,001 \\
Higiene dos banheiros adequada & 91,18 & 100 & 80 & 90,91 & 100 & 78,57 & 100 & 63,41 & 0,002 \\
Espaço para locomoção na sala de aula & 82,35 & 100 & 72 & 54,55 & 66,67 & 71,43 & 81,25 & 41,46 & 0,005 \\
Presença de poeira & 70,59 & 20 & 48 & 90,91 & 78,95 & 71,43 & 50 & 0 & $<0,001$ \\
Sala de aula adequada ao no de alunos & 67,65 & 100 & 68 & 54,55 & 68,42 & 64,29 & 75 & 36,59 & $<0,001$ \\
Limpeza satisfatória & 67,65 & 100 & 56 & 81,82 & 100 & 50 & 100 & 51,22 & 0,001 \\
Presença de ruído & 64,71 & 40 & 72 & 90,91 & 89,47 & 64,29 & 25 & 60,98 & 0,002 \\
Acústica da sala satisfatória & 64,71 & 80 & 48 & 63,64 & 68,42 & 35,71 & 81,25 & 56,1 & - \\
Comprometimento com a manutenção da escola & 64,71 & 100 & 60 & 90,91 & 94,74 & 64,29 & 81,25 & 60,98 & 0,033 \\
Temperatura adequada & 64,71 & 100 & 36 & 54,55 & 31,58 & 50 & 68,75 & 39 & $<0,001$ \\
Móveis adequados a estatura dos professores & 61,76 & 100 & 80 & 54,55 & 78,95 & 71,43 & 75 & 63,41 & - \\
Utilização de produtos químicos & 52,94 & 0 & 16 & 9 & 36,84 & 28,57 & 18,75 & 29,27 & - \\
Presença de eco & 26,47 & 0 & 16 & 18,18 & 15,79 & 28,57 & 12,5 & 2,44 & - \\
Presença de fumaça & 23,53 & 40 & 16 & 54,55 & 47,37 & 28,57 & 43,75 & 51,22 & - \\
Umidade & 11,76 & 0 & 8 & 0 & 15,79 & 14,29 & 31,25 & 9,76 & - \\
\hline
\end{tabular}

Legenda: SE - Significância estatística

Tabela 3 - Comparação entre as unidades quanto à referência à alteração vocal

\begin{tabular}{ccccccccc}
\hline \multirow{2}{*}{ Alteração Vocal } & \multicolumn{7}{c}{ Unidades Escolares/ Dados em \% } \\
\cline { 2 - 9 } & I & II & IV & V & VI & VII & VIII & IX \\
\hline & 76,47 & $40 \%$ & $40 \%$ & $63,64 \%$ & $73,68 \%$ & $64,29 \%$ & $75 \%$ & $70,73 \%$ \\
\hline
\end{tabular}

Tabela 4 - Comparação entre as unidades quanto às causas da alteração vocal

\begin{tabular}{lcccccccccc}
\hline \multirow{2}{*}{ Causa do problema } & \multicolumn{1}{c}{ Unidades Escolares/ Dados em \% } & \multirow{2}{*}{ SE } \\
\cline { 2 - 9 } & I & II & IV & V & VI & VII & VIII & IX & - \\
\hline Uso intensivo da voz & 96,15 & 100 & 100 & 85,71 & 85,71 & 100 & 100 & 89,66 & - \\
Estresse & 61,54 & 100 & 50 & 57,14 & 71,43 & 66,67 & 66,67 & 55,17 & - \\
Alergia & 57,69 & 50 & 30 & 85,71 & 35,71 & 66,67 & 66,67 & 62 & - \\
Exposição ao barulho & 56 & 100 & 40 & 28,57 & 61,54 & 44,44 & 33,33 & 37,93 & - \\
Exposição ao frio & 42,31 & 0 & 10 & 71,43 & 28,57 & 33,33 & 25 & 27,59 & - \\
Infecção Respiratória & 30,77 & 50 & 30 & 85,71 & 28,57 & 11,11 & 25 & 13,79 & 0,021 \\
Gripe Constante & 23 & 50 & 10 & 28,57 & 14,29 & 0 & 16,67 & 13,79 & - & - \\
Sem razão aparente & 7,69 & 0 & 0 & 0 & 14,29 & 11,11 & 0 & 6,9 & - \\
\hline
\end{tabular}

Legenda: SE - Significância estatística 
Tabela 5 - Comparação entre as unidades no quesito saúde segundo os professores

\begin{tabular}{|c|c|c|c|c|c|c|c|c|c|}
\hline \multirow{2}{*}{ Queixa } & \multicolumn{8}{|c|}{ Unidades Escolares/ Dados em \% } & \multirow{2}{*}{ SE } \\
\hline & $\mathbf{I}$ & II & IV & $\mathbf{v}$ & VI & VII & VIII & IX & \\
\hline Ansiedade & 70,59 & 60 & 84 & 54,55 & 63,16 & 57,14 & 75 & 65,85 & - \\
\hline Incomodo a ruídos & 67,65 & 0 & 60 & 45,45 & 63,16 & 57,14 & 43,75 & 36,59 & 0,036 \\
\hline Alergias & 50 & 60 & 68 & 45,45 & 57,89 & 64,29 & 56,25 & 58,54 & - \\
\hline Dores de cabeça & 50 & 40 & 40 & 54,55 & 68,42 & 57,14 & 43,75 & 73,17 & - \\
\hline Ganho de peso & 41,18 & 40 & 44 & 63,64 & 42,11 & 42,86 & 25 & 36,59 & - \\
\hline Doenças de Vias aéreas & 38,24 & 60 & 32 & 72,73 & 31,58 & 35,71 & 31,25 & 24,39 & - \\
\hline Resfriados freqüentes & 39,39 & 40 & 12 & 27,27 & 26,32 & 28,57 & 18,75 & 39 & - \\
\hline Dores no Corpo & 35,29 & 40 & 48 & 27,27 & 47,37 & 64,29 & 43,75 & 39 & - \\
\hline Presença de zumbido & 29,41 & 0 & 24 & 9 & 21 & 14,29 & 25 & 29,27 & - \\
\hline Azia & 29,41 & 20 & 32 & 45,45 & 15,79 & 21,43 & 0 & 31,71 & - \\
\hline Alteração da audição & 26,47 & 0 & 44 & 18,18 & 36,84 & 14,29 & 18,75 & 34,15 & - \\
\hline Problemas na coluna & 26,47 & 0 & 48 & 36,36 & 57,89 & 42,86 & 43,75 & 41,46 & - \\
\hline $\begin{array}{l}\text { Presença de } \\
\text { tontura/vertigem }\end{array}$ & 23,53 & 20 & 32 & 36,36 & 31,58 & 0 & 31,25 & 29,27 & - \\
\hline Depressão & 17,65 & 0 & 24 & 18,18 & 21 & 21,43 & 25 & 14,63 & - \\
\hline Gastrite & 14,71 & 20 & 20 & 18,18 & 5,26 & 14,29 & 0 & 26,83 & - \\
\hline
\end{tabular}

Legenda: SE - Significância estatística

\section{DISCUSSÃO}

Caracterizar os riscos ocupacionais presentes na escola e sua interferência sobre a saúde e voz de professores mostra-se apropriado, uma vez que permite planejar e desenvolver ações que favoreçam um ambiente saudável para se viver e que promovam a qualidade de vida de todos os segmentos que lá estudam e trabalham.

Os dados sobre as peculiaridades das unidades da rede municipal de ensino analisados mostraram que a única diferença entre elas foi o número de homens que trabalham na unidade II. Comprovase que as mulheres ocupam os postos de trabalho na docência em todos os níveis de ensino nacional e internacionalmente, especialmente na educação básica e média, sendo esse dado compatível com outros estudos ${ }^{13-15}$.

Em relação aos riscos ocupacionais decorrentes da organização de trabalho, a Tabela 1 mostra que os pontos comuns entre as unidades foram: o tempo curto para desenvolver as atividades dentro da escola, avaliado como negativo, enquanto que a autonomia quanto ao planejamento foi valorado como positivo pelos docentes.

Tempo insuficiente de desenvolver as atividades dentro da escola indica que os docentes possuem uma alta demanda de atividades no trabalho, tendo que preparar ou completar as atividades fora do mesmo, dados reiterados em outros autores ${ }^{9,16}$. Esse excesso de trabalho contribui para a diminuição de momentos de lazer e para o aparecimento do estresse físico e psicológico com prejuízo para a saúde e desempenho profissional do docente ${ }^{17,18}$. Já a falta de autonomia sobre o próprio trabalho pode trazer para os docentes sentimentos de insatisfação e estresse e é considerado um fator de risco para a saúde dos professores, porém nesse estudo isto não foi verificado, contrariamente a literatura ${ }^{16}$.

Alguns aspectos quanto à organização de trabalho mostraram-se desfavoráveis aos professores do presente estudo, como a ausência de lugar para descanso, fiscalização do desempenho e ambiente de trabalho estressante. Alguns autores também constataram a ausência de lugar para descanso/ repouso e os prejuízos que isso causa a saúde dos professores, enquanto outros observaram que $85,4 \%$ dos docentes de sua pesquisa consideravam o ambiente de trabalho não calmo ${ }^{9,19}$.

Os fatores de risco presentes no ambiente de trabalho (Tabela 2) mostraram frequências diversas, no entanto, alguns estiveram sempre presentes nas referências dos professores, como o ruído, a fumaça e acústica insatisfatória.

É recorrente nas pesquisas realizadas em escolas, a menção dos altos níveis de ruído nelas presentes e suas consequências negativas para a saúde e para o processo ensino-aprendizagem 1,4,20,21. Outro fator nocivo é a presença de fumaça, pois a sua exposição constante pode acarretar doenças de vias respiratórias e quadros alérgicos como bronquite, asma e rinite ${ }^{9,22}$.

A unidade escolar IX apresentou maior frequência de queixas em relação à sala de aula inadequada ao número de alunos $(63,41 \%)$ e consequente espaço insuficiente para locomoção $(58,54 \%)$, fatores que, na literatura, ora estiveram associados a frequência de disfonia em professores ${ }^{23}$ ora não ${ }^{13}$. Quanto aos aspectos vocais, a menção à presença de alteração vocal alcançou um máximo de $76,47 \%$ na unidade I e um mínimo de $40 \%$ nas unidades II e IV, ambas avaliadas 
como muito altas e muito acima do desejável, considerando-se que o professor tem em sua voz seu principal instrumento de trabalho. Estes dados vêm ao encontro da literatura que mostra também alto índice de alteração vocal nos professores ${ }^{13,24}$.

Alguns fatores sempre foram mencionados pelos docentes quando se abordou a possível etiologia das alterações vocais auto-referidas, como: uso intensivo de voz, estresse, exposição ao barulho, alergia, infecção respiratória. Na unidade I, 57,69\% dos docentes assinalaram a alergia como causa das alterações vocais e o fator que pode estar relacionado a isso é a utilização de produtos químicos, considerando-se que esta escola mostrou o maior número de queixas de uso desses produtos para limpeza $(54,94 \%)$. Já na unidade $\mathrm{V}$ a alergia dos professores $(85,71 \%)$ pode estar relacionada ao nível elevado de poeira $(90,91 \%)$ na escola. A literatura mostra que os professores possuem maiores chances de apresentar alergias e problemas respiratórios, assim como constata que existe uma diferença significativa entre ocorrência de disfonia e alergia ${ }^{7}$.

Em relação à saúde, a ansiedade, alergias e dores de cabeça foram queixas comuns e em alta porcentagem entre os docentes (Tabela 5). Outros estudos também destacaram cefaleia e ansiedade como sintomas mais frequentes ${ }^{2}$. Neste item, ainda houve variação de porcentagens entre as unidades, indicando que um fator pode ser um problema de saúde para alguns professores e não para outros. Especificamente em relação às consequências à saúde do professor ressalta-se que há mais queixas físicas que emocionais, indicando que o corpo do professor é o maior alvo das condições adversas de trabalho.
Pelo exposto, as condições referentes ao ambiente físico se mostraram em maior número e estatisticamente significantes, que aquelas pertinentes à organização de trabalho. Cabe lembrar, no entanto, que os fatores de risco de ambas as fontes se articulam e podem interferir na saúde e voz dos professores. Apesar disso, comparados com outras pesquisas, os achados desse estudo, indicam que as unidades de ensino avaliadas apresentam boas condições de trabalho, mesmo que não ideais ${ }^{9}$.

Merece menção ao fato de que, embora haja condições satisfatórias do ambiente físico, o estresse referido por grande parte do corpo docente parece advir de outras causas, as quais requerem detalhamento por meio de novas investigações.

\section{CONCLUSÃO}

Há riscos ocupacionais relacionados à organização e ambiente de trabalho, que podem prejudicar a voz e a saúde dos docentes, com especificidades para cada unidade escolar decorrentes da localização geográfica da escola, arquitetura e materiais empregados na construção, nível de conservação e melhorias realizadas, assim como características da organização do trabalho que lá se estabelecem. Professores de todas as unidades referiram altos índices de alteração vocal e referência a doenças. As escolas exibem pontos comuns, sendo o tempo restrito para desenvolver todas as atividades o mais evidente. $\mathrm{O}$ desvelamento das relações entre trabalho, saúde e voz nas diferentes escolas da rede municipal subsidiará o gestor na implantação de ações contextualizadas para promover ambiente saudável para a comunidade escolar.

\begin{abstract}
Purpose: to compare the work, health and voice conditions of teachers from different city schools. Methods: 165 teachers took part, mean age 37, married, predominance of women and university degree, from the city school system of a city from the state of São Paulo, who answered to the questionnaire proposed by Ferreira et al (2003), covering socio-demographic topics, functional situation, vocal and health aspects. The teachers' answers were separated by scholar unit and submitted to a statistical analysis. Results: there was a significant difference among schools as for the presence of restrooms, constant surveillance and the ease of leaving the classroom, number of students per classroom $(<0.001)$, noise $(0.002)$, lightning $(<0.001)$, cleaning $(0.001)$, toilets' hygiene $(0.002)$, dust $(0.001)$, classroom size $(0.020)$, space sufficiency in classrooms $(0.033)$ and temperature $(<0.001)$. The mentioning of vocal alteration varied from $76.47 \%$ to $40 \%$, with no significance for teachers from different schools. The health problems were diversified with significant difference only to sound nuisance (0.036). Conclusion: there are specific occupational risks related to organizational and work environment in distinct scholar units that interfere in the docents' voice and health. By having this information the manager can implement contextualized initiatives in order to promote a healthy environment for all the scholar community.
\end{abstract}

KEYWORDS: Voice Disorders; Faculty; Occupational Risks 


\section{REFERÊNCIAS}

1. Skarlatos D, Manatakis M. Effects of classroom noise on students and teachers in Greece. Percept Mot Skills. 2003; 96(2):539-44.

2. Ortiz E, Alves LEA, Costa E. Saúde vocal de professores da rede municipal de ensino de cidade do interior de São Paulo. Rev Bras Méd Trabalho. 2004; 2(4):263-6.

3. Porto LA, Reis IC, Andrade JM, Nascimento CR, Carvalho FM. Doenças ocupacionais em professores atendidos pelo Centro de Estudos da Saúde do Trabalhador (CESAT). Rev Baiana Saúde Pública. 2004; 28(1):33-49.

4. Dreossi RCF, Momensohn-Santos T. O ruído e sua interferência sobre estudantes em uma sala de aula: revisão de literatura. Pró-Fono. 2005; 17(2):251-8.

5. Simberg S, Sala E, Vehmas K, Laine A. Changes in the prevalence of vocal symptoms among teachers during a twelve-year period. J Voice. 2005; 19(1):95-102.

6. Thibeault SL, Merrill RM, Roy N, Gray SD, Smith EM. Occupational risk factors associated with voice disorders among teachers. Ann Epidemiol. 2004; 14(10):786-92.

7. Roy N, Merrill RM, Thibeault S, Gray SD, Smith $\mathrm{EM}$. Voice disorders in teachers and the general population: effects on work performance, attendance and future career choices. J Speech Lang Hear Res. 2004; 47(3):542-51.

8. Roy N, Merrill RM, Thibeault S, Parsa RA, Gray SD, Smith EM. Prevalence of voice disorders in teachers and the general population. J Speech Lang Hear Res. 2004; 47(2): 281-93.

9. Ferreira LP, Giannini SPP, Figueira S, Silva EE, Karmann DF, Souza TMT. Condições de produção vocal de professores da prefeitura do município de São Paulo. Dist Comun. 2003; 14(2):275-307.

10. Secretaria de Estado de Saúde de São Paulo. Distúrbios da voz relacionados ao trabalho. Boletim Epidemiológico Paulista. 2006; 3(26):16-22 [acesso em 18 dez 2007] Disponível em: http://www.cve. saude.sp.gov.br/agencia/bepa26_dist.htm.

DOI: 10.1590/S1516-18462009005000061

RECEBIDO EM: 08/11/2008

ACEITO EM: 30/06/2009

Endereço para correspondência:

Emilse Aparecida Merlin Servilha

Av. John Boyd Dunlop, s/no

Campinas - SP

CEP: 13060-904

E-mail: emilsemerlin@uol.com.br
11. Rogerson J, Dodd B. Is there an effect of dysphonic teachers' voices on children's processing of spoken language? J Voice. 2005; 19(1):47-60.

12. Rosner B. Fundamentals of biostatistics. 2. ed. Boston: PWS Publishers; 1986. 584p.

13. Schwarz K, Cielo CA. A voz e as condições de trabalho de professores de cidades pequenas do Rio Grande do Sul. Rev Soc Bras Fonoaudiol. 2005; 10(2):83-90.

14. Preciado J, Pérez C, Calzada $M$, Preciado $P$. [Prevalence and incidence studies of voice disorders among teaching staff of La Rioja, Spain. Clinical study: questionnaire, function vocal examination, acoustic analysis and videolaryngostroboscopy]. Acta Otorrinolaringol Esp. 2005; 56(5):202-10.

15. Kasama ST, Brasolotto AG. Percepção vocal e qualidade de vida. Pró-Fono. 2007; 19(1):19-28.

16. Delcor NS, Araújo TM, Reis EJFB, Porto LA, Carvalho FM, Silva MO, et al. Condições de trabalho e saúde dos professores da rede particular de ensino de Vitória da Conquista, Bahia, Brasil. Cad Saúde Pública. 2004; 20(1):187-96.

17. Reis EJ, Araújo TM, Carvalho FM, Barbalho L, Silva MO. Docência e exaustão emocional. Educ Soc. 2006; 27(94):229-53.

18. Servilha EAM. Estresse em professores universitários na área de fonoaudiologia. Rev Ciênc Méd. 2005; 14(1):43-52.

19. Araújo TM, Sena IP, Viana MA, Araújo EM. Malestar docente: avaliação de condições de trabalho e saúde em uma instituição de ensino superior. Rev Baiana Saúde Pública. 2005; 29(1):6-21.

20. Salas SWA, Centeno HJ, Landa CE, Amaya CJM, Benites GMR. Prevalencia de disfonía en profesores del distrito de Pampas - Tayacaja Huancavelica. Rev Med Hered. 2004; 15(3):125-30. 21. Grillo MHMM, Penteado RZ. Impacto da voz na qualidade de vida de professore(a)s do ensino fundamental. Pró-Fono. 2005; 17(3):321-30.

22. Giannini SPP, Passos MC. Histórias que fazem sentidos: as determinações das alterações vocais do professor. Dist Comun. 2006; 18(2):245-57.

23. Fuess VLR, Lorenz MC. Disfonia em professores do ensino municipal: prevalência e fatores de risco. Rev Bras Otorrinolaringol. 2003; 69(6):807-12.

24. Troni CR, Arakaki FN, Lima FS, Mott L, Rodrigues KA, Ferreira LP. Professores em contexto profissional e não profissional: análise objetiva e subjetiva dos aspectos da articulação e da postura. Dist Comun. 2006; 18(2):179-88. 